\title{
BAG3 increases the invasiveness of uterine corpus carcinoma cells by suppressing miR-29b and enhancing MMP2 expression
}

\author{
SHUTARO HABATA, MASAHIRO IWASAKI, ASUKA SUGIO, MIWA SUZUKI, MASATO TAMATE, \\ SEIRO SATOHISA, RYOICHI TANAKA and TSUYOSHI SAITO
}

Department of Obstetrics and Gynecology, Sapporo Medical University, Sapporo, Japan

Received December 25, 2014; Accepted February 17, 2015

DOI: $10.3892 /$ or.2015.3831

\begin{abstract}
Approximately $30 \%$ of uterine corpus carcinomas are diagnosed at an advanced stage and have a poor prognosis. Our previous study indicated that BCL2-associated athanogene 3 (BAG3) enhances matrix metalloproteinase-2 (MMP2) expression and binds to MMP2 to positively regulate the process of cell invasion in ovarian cancer cells. Recently, altered miRNA expression patterns were observed in several groups of patients with endometrial cancers. One of the altered miRNAs, miR-29b, reportedly reduces tumor invasiveness by suppressing MMP2 expression. Our aim in the present study was to examine the relationships among BAG3, miR-29b and MMP2 in endometrioid adenocarcinoma cells. We found that BAG3 suppresses miR-29b expression and enhances MMP2 expression, which in turn increases cell motility and invasiveness. Moreover, restoration of miR-29b through BAG3 knockdown reduced MMP2 expression, as well as cell motility and invasiveness. Collectively, our findings indicate that BAG3 enhances MMP2 expression by suppressing miR-29b, thereby increasing the metastatic potential of endometrioid adenocarcinomas.
\end{abstract}

\section{Introduction}

Cancer of the uterine corpus is the second most commonly diagnosed cancer among women in the USA, following breast cancer. Although the majority of endometrial cancers $(68 \%)$ are detected at an early stage (stage I-II), 32\% of the patients have regional or distant metastasis at diagnosis (1). Endometrioid adenocarcinoma (EAC) accounts for threequarters of all uterine corpus cancers. Most primary EACs are considered to be of low risk and are usually treatable. Frequently, however, their potential to recur and metastasize

Correspondence to: Dr Masahiro Iwasaki, Department of Obstetrics and Gynecology, Sapporo Medical University, South 1 West 16, Chuo-ku, Sapporo, Hokkaido 060-8543, Japan

E-mail: miwasaki@sapmed.ac.jp

Key words: BAG3, MMP2, uterine corpus carcinoma, miR-29b, cell invasion leads to a poor prognosis with significant mortality (2). It is therefore necessary to identify novel therapeutic targets and treatment strategies, both of which may be obtained through an increased understanding of the molecular mechanisms involved in EACs.

BAG3 is one of the six BAG family proteins, which are involved in such cellular processes as proliferation, apoptosis, adhesion and migration. BAG3 contains a BAG domain, a WW domain near its $\mathrm{N}$-terminus and a proline-rich region (multiple PXXP motifs) $(3,4)$. The BAG domain binds to the ATPase domain of the molecular chaperone HSP70, where it acts as a nucleotide exchange factor (5). The WW domains act as protein interaction modules that bind a proline-rich motif, XPPXY. Recognition of their peptide ligands by some WW domains is phosphorylation-dependent, and WW domains have been identified in several signal transduction proteins that interact with plasma membrane receptor complexes or with components of the submembranous cytoskeleton $(3,4)$. Proline-rich regions act by binding SH3 ligand motifs (6). Previous studies have demonstrated that BAG3 is overexpressed in several epithelial cancers, mainly adenocarcinomas (7). In addition, a previous study from our group showed that BAG3 induced MMP2 expression and then bound to MMP2 to positively regulate the process of cell invasion (8). However, little is known about the role of BAG3 in EACs.

MicroRNAs (miRNAs) are small non-coding RNAs that function as negative regulators of gene expression by targeting mRNAs based on their complementarity to the mRNA 3' untranslated region (3'-UTR) (9). A growing body of evidence indicates that a disordered expression of miRNA contributes to the initiation and progression of human cancers. It has been proven, for example, that miRNAs participate in human carcinogenesis by acting as either tumor suppressors or oncogenes (10). In addition, because miRNAs exhibit tissue-specific expression patterns (11), they may be of value serving as therapeutic targets for cancer treatment and/or as biomarkers.

Recently, changes in the patterns of miRNA expression were observed in endometrial cancer specimens collected from several study cohorts $(11,12)$. In both EAC and serous adenocarcinoma, numerous miRNAs are overexpressed or underexpressed, as compared to healthy endometrium. In serous endometrial carcinoma, for example, the levels of miR-10b, $-29 b$ and $-455-5 p$ are all reduced, and this effect 
appears to be involved in the cancer progression (13). Among these molecules, miR-29b reportedly acts as a tumor suppressor in various cancers, including acute myeloid leukemia, cholangiocarcinoma and hepatocellular carcinoma, by regulating tumor apoptosis, invasion and metastasis through its targeting of myeloid cell leukemia 1 (Mcl-1) (14-16) and matrix metalloproteinase 2 (MMP-2) (17). In the present study, we explored the relationship between BAG3 and miR29b in cultured EAC cells. Using the Ishikawa (grade 1-derived) and HEC108 (grade 3-derived) EAC cell lines, we assessed the expression of BAG3, MMP2 and miR29-b, and evaluated their effects on cell motility and invasiveness. Our findings have furthered our understanding of the function and mechanism of BAG3 and miR-29b in EACs.

\section{Materials and methods}

Cells and cell culture. Two established uterine EAC cell lines were used in this study. The Ishikawa line was established from a grade 1 cancer, while the HEC108 line was established from a grade 3 cancer. Ishikawa cells were cultured in RPMI-1640 (Gibco, Grand Island, NY, USA) supplemented with $10 \%$ fetal bovine serum (Hyclone, Logan, UT, USA) and $1 \%$ penicillin/streptomycin (Gibco). HEC108 cells were cultured in Dulbecco's modified Eagle's medium (DMEM) (Gibco) supplemented with $10 \%$ FBS and $1 \%$ penicillin/streptomycin. Both cell lines were maintained in a $\mathrm{CO}_{2}$ incubator $\left(5 \% \mathrm{CO}_{2}\right)$ at $37^{\circ} \mathrm{C}$.

Gene silencing using a short hairpin RNA (shRNA) vector. A gene silencing vector (pLTRH1) containing a RNA polymerase promoter producing shRNA specific for BAG3 was used for transfection. Oligonucleotides [5'-GATCCCGTACCTGATG ATCGAAGAGTTTCAAGAGAACTCTTCGATCATCAGGT ATTTTTGGAG-3' (sense) and 5'-TCGACTTCCAAAAAAT ACCTGATGATCGAAGAGTTCTCTTGAAACTCTTCGAT CATCAGGTACGG-3' (antisense)] specific for mouse bag3 were synthesized and subcloned into the $B g l \mathrm{II}$ and $\mathrm{SalI}$ sites, downstream of the H1 promoter (18). G3T-hi amphotrophic packaging cells (Takara Bio, Shiga, Japan) were transfected with pLTRH1bag3 puro or empty pLTRH1 puro vector according to the manufacturer's instructions to obtain a retroviral supernatant, which was added at a 1:5 ratio to DMEM supplemented with $10 \%$ FBS and then used to infect Ishikawa or HEC108 cells. Infected cells were then selected by incubation with $1.0 \mu \mathrm{g} / \mathrm{ml}$ puromycin (Gibco) for $48 \mathrm{~h}$ after infection. High-responder clones to BAG3 knockdown were selected for subsequent experiments.

Real-time quantitative reverse transcription PCR ( $q R T-P C R)$ for $m R N A$. Total RNA was extracted from cells using TRIzol reagent (Invitrogen, Carlsbad, CA, USA), after which cDNA was synthesized from $1 \mu \mathrm{g}$ of RNA using VILO master mix (Invitrogen). qRT-PCR was carried out using Fast SYBR Green Master Mix (Applied Biosystems, Foster, CA, USA) in a StepOnePlus ${ }^{\mathrm{TM}}$ Real-Time PCR system (Applied Biosystems). mRNA levels were standardized to the level of glyceraldehyde 3-phosphate dehydrogenase (GAPDH) mRNA. The PCR protocol entailed denaturation at $95^{\circ} \mathrm{C}$ for $10 \mathrm{~min}$ followed by 40 cycles of $95^{\circ} \mathrm{C}$ for $15 \mathrm{sec}$ and $60^{\circ} \mathrm{C}$ for $60 \mathrm{sec}$. The following primers were designed and used for qRT-PCR: for BAG3, 5'-TGAGAAGTTTAACCCCGTTGCTTGT-3' (forward) and 5'-CCCCATCTACCCCTCCAGTCCAG-3' (reverse); for MMP2, 5'-ACCTGGATGCCGTCGTGGAC-3' (forward) and 5'-TGTGGCAGCACCAGGGCAGC-3' (reverse); for GAPDH, 5'-TGAACGGGAAGCTCACTGG-3' (forward) and 5'-TCC ACCACCCTGTTGCTGTA-3' (reverse). Gene expression was calculated using the $2^{-\Delta \mathrm{Ct}}$ method.

$q R T-P C R$ for microRNA. Total RNA was extracted using TRIzol reagent (Invitrogen), after which reverse transcription was performed with $10 \mathrm{ng}$ of total RNA using a TaqMan ${ }^{\circledR}$ MicroRNA Reverse Transcription kit (Applied Biosystems) and sequence-specific RT primers from the TaqMan MicroRNA assays (Applied Biosystems) according to the manufacturer's instructions. Separate reverse transcription reactions were run for each TaqMan MicroRNA assay on each RNA sample. qRT-PCR was performed with cDNA using inventoried TaqMan MicroRNA assays and TaqMan Universal Master Mix II (Applied Biosystems). The assay was performed in triplicate, and the PCR amplification was performed using a StepOnePlus $^{\mathrm{TM}}$ Real-Time PCR system. Gene expression was calculated using the $2^{-\Delta \mathrm{Ct}}$ method.

Lysate production. Cell lysates were produced from subconfluent cell cultures. After scraping the cells from the dishes, they were placed in RIPA buffer [50 mM Tris- $\mathrm{HCl}(\mathrm{pH} 8.0)$, $150 \mathrm{mM} \mathrm{NaCl}, 0.1 \% \mathrm{SDS}, 1 \% \mathrm{NP} 40$ and $0.5 \%$ sodium deoxycholate] containing a protease inhibitor cocktail (1:100 dilution; Thermo Scientific, Rockford, IL, USA). The cells were then lysed by sonication, after which the lysates were centrifuged at $15,000 \mathrm{rpm}$ for $15 \mathrm{~min}$ at $4^{\circ} \mathrm{C}$ to pellet the nuclei. The supernatant was then collected as the cell lysate.

Western blotting. After measuring the protein content, lysates were diluted in $2 \mathrm{X}$ sample buffer [0.5 M Tris- $\mathrm{HCl}$ ( $\mathrm{pH} 6.8$ ), $10 \%$ SDS, $\beta$-mercaptoethanol and $1 \%$ BPB] and boiled for $5 \mathrm{~min}$ at $100^{\circ} \mathrm{C}$. Samples containing $20 \mu \mathrm{g}$ of protein were then electrophoresed (200 V for $35 \mathrm{~min}$ ) on $12 \%$ SDS polyacrylamide gel, after which the separated proteins were transferred onto PVDF membranes. After being blocked with 5\% non-fat dry milk in TBS [10 mM sodium phosphate (pH 7.8), $150 \mathrm{mM}$ $\mathrm{NaCl}$ and $0.05 \%$ Tween-20], the membrane was probed with the following primary antibodies: rabbit monoclonal antibodies against BAG3 (1:1,000 dilution; gift of Dr S. Takayama), mouse monoclonal antibodies against hMMP2 (1:150 dilution; F-68; Daiichi Fine Chemical Co., Ltd., Toyama, Japan) and mouse monoclonal antibodies against $\beta$-actin $(1: 5,000$ dilution; A5441; Sigma-Aldritch, St. Louis, MO, USA). After being washed with PBS-T, the membranes were incubated with secondary horseradish peroxidase-conjugated antibodies. The protein was visualized using ECL Prime Western Blotting Detection Reagent and ImageQuant LAS 500 (GE Healthcare, Buckinghamshire, UK).

Cell motility and invasion assays. BAG3 knockdown cells and miR29b mimic/inhibitor transfectants were used for motility and invasion assays. To assess motility, the cells were seeded into the upper chambers of the 24-well Transwell chambers (Corning, Inc., Corning, NY, USA) at the density of $5 \times 10^{4}$ cells 
A

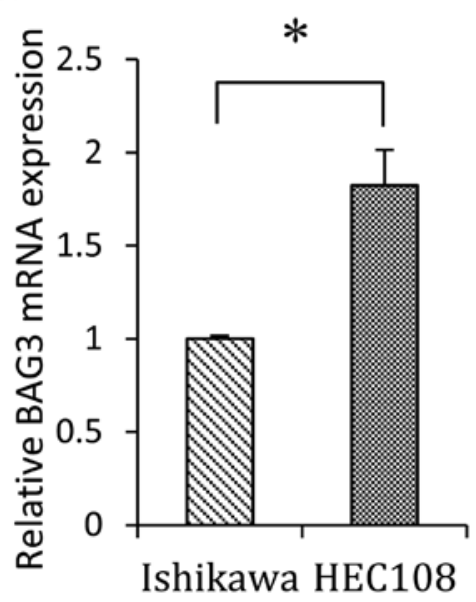

B

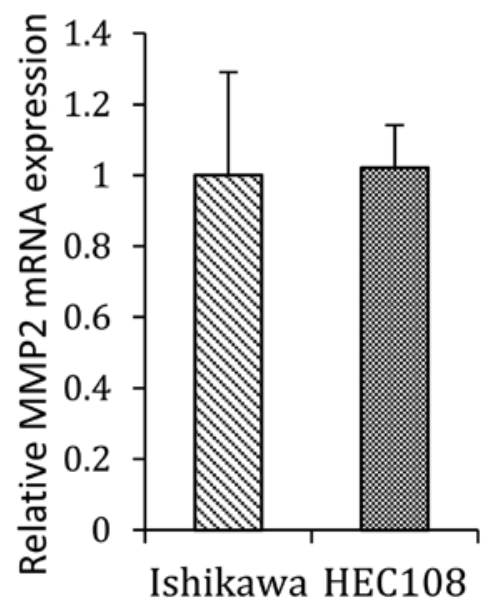

C

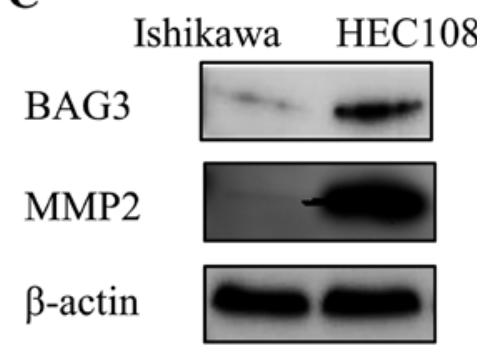

Figure 1. Western blot and real-time PCR analyses of BAG3 and MMP2 expression in EAC cells. (A and B) Levels of BAG3 (A) and MMP-2 (B) mRNA in Ishikawa and HEC108 cells were determined by using real-time qRT-PCR. Bars depict the relative mRNA levels normalized to the level of GAPDH mRNA. The results are presented as means $\pm \mathrm{SD} ;{ }^{*} \mathrm{P}<0.05$. (C) Western blot analysis of BAG3 and MMP-2 expression in Ishikawa and HEC108 cells. Blots were probed using rabbit polyclonal anti-BAG3 or anti-MMP2 antibody. As a loading control, the blots were reprobed using mouse monoclonal anti-actin antibody.

per well and cultured in medium containing $0.5 \%$ BSA. Medium containing $10 \% \mathrm{FBS}$ and $10 \mu \mathrm{g} / \mathrm{ml}$ of fibronectin was added to the lower chamber. The plates were then incubated for $7 \mathrm{~h}$ at $37^{\circ} \mathrm{C}$, after which the cells that remained on top of the membrane were removed with a cotton swab. The cells that had migrated through the membrane were fixed in formalin, stained with hematoxylin and eosin (H\&E) and counted in five 20x microscope (Olympus DP70) (Olympus, Tokyo, Japan) fields. To assess invasion, cells were incubated for $60 \mathrm{~h}$ at $37^{\circ} \mathrm{C}$ in Corning ${ }^{\circledR}$ BioCoat ${ }^{\mathrm{TM}}$ Matrigel $^{\circledR}$ Invasion Chambers with 8.0- $\mu \mathrm{m}$ pores (Discovery Labware, Inc., Bedford, MA, USA). The data are from three independent experiments.

Mature miRNA transfection. Using Lipofectamine RNAiMAX (Invitrogen), cells grown in 6-cm dishes were transfected with miRNA-29b mirVana miRNA mimic (Ambion, Austin, TX, USA) to augment miR-29b activity, or with an inactive negative control for miRNA-29b mirVana miRNA mimic (Ambion). Alternatively, they were transfected with a miRNA-29b mirVana miRNA inhibitor (Ambion) to diminish miR-29b activity or a negative control for a miRNA-29b mirVana miRNA inhibitor (Ambion).

Luciferase assay. HEC108 cells cultured in 96-well plates were co-transfected using $17 \mathrm{nM}$ miR-29b mimic or its control plus $25 \mathrm{ng}$ of the 3'-UTR of MMP2 mRNA, which contained the target sites for miR-29b, or empty vector. The cells were collected $48 \mathrm{~h}$ after transfection and analyzed using a LightSwitch Assay System (Switch Gear Genomics, Menlo Park, CA, USA). Luciferase activity was detected using an Infinite M 1000 PRO microplate reader (Tecan Group Ltd., Männedorf, Switzerland). All the experiments were performed in triplicate.

Statistical analysis. Student's t-tests were used for statistical evaluation of the data. Values of $\mathrm{P}<0.05$ were considered significant. SPSS 22.0 (IBM, Armonk, NY, USA) was used in the analysis.

\section{Results}

Expression of BAG3 and MMP2 in EAC cell lines. Ishikawa and HEC108 cells were used for western blot and qRT-PCR analyses. There was little difference in MMP2 mRNA expression between the two cell lines (Fig. 1B), but expression of MMP2 protein was detected only in HEC108 cells (Fig. 1C). Similarly, BAG3 mRNA and protein were more strongly expressed in HEC108, than Ishikawa cells (Fig. 1A and C).

BAG3 knockdown reduces MMP2 expression. To determine the effect of reducing BAG3 expression on MMP2 activity, Ishikawa and HEC108 cells were infected with a retroviral 
Ishikawa

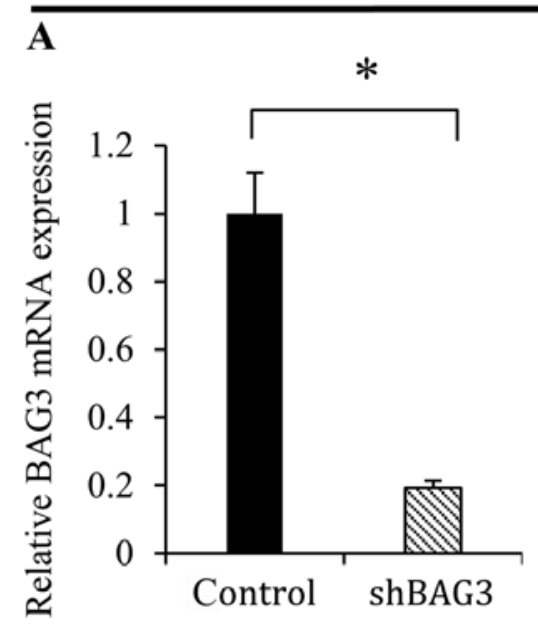

B

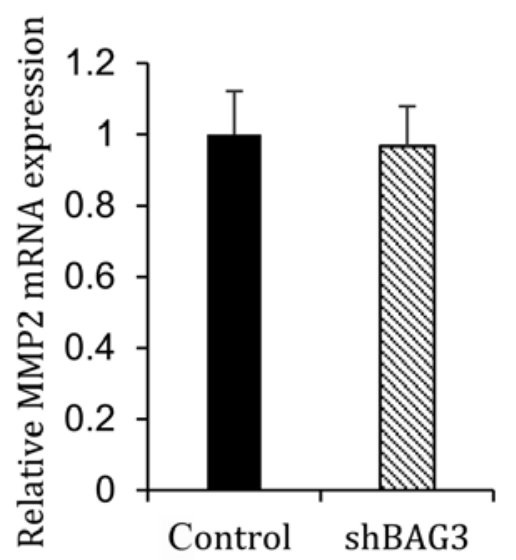

C

\section{Control shBAG3}

BAG3

MMP2

$\beta$-actin

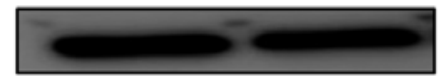

HEC108

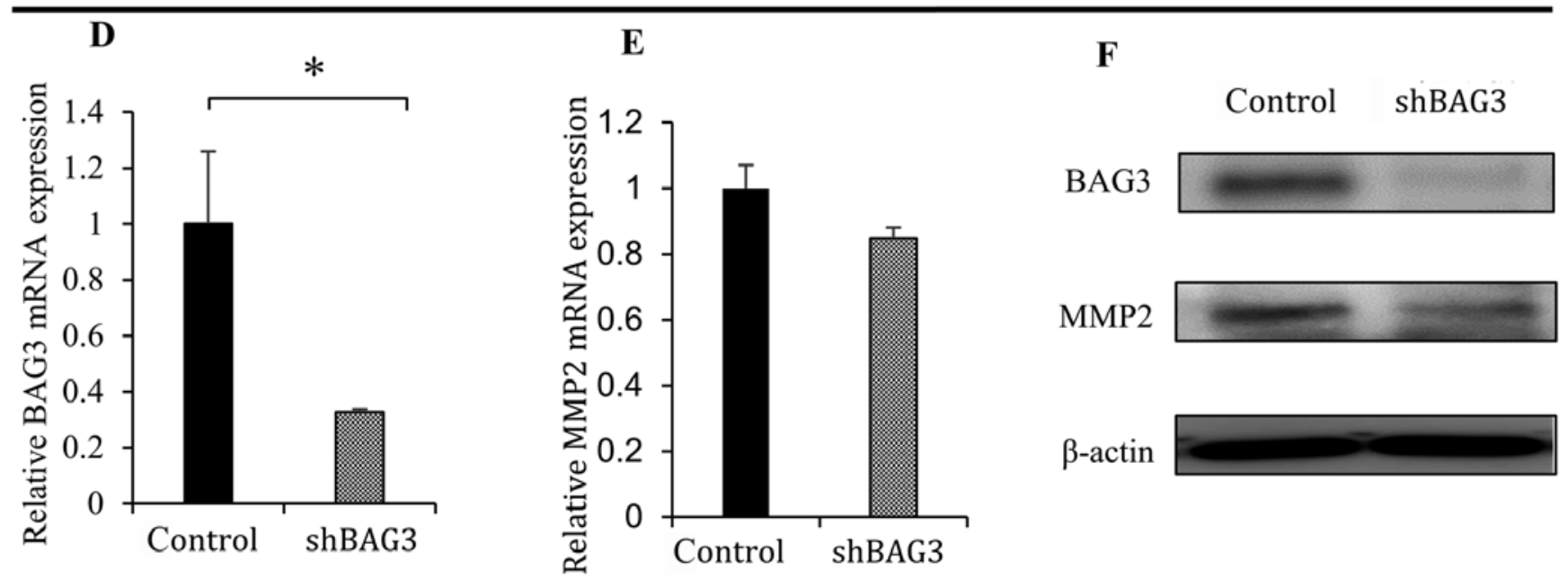

Figure 2. Effect of BAG3 knockdown on MMP2 levels. (A, B, D and E) Ishikawa (A and B) and HEC108 (D and E) cells were infected with either a control (LTRH) or shBAG3 (BAG3 knockdown) vector. BAG3 (A and D) and MMP-2 (B and E) mRNA levels were analyzed using real-time qRT-PCR. Bars depict relative mRNA levels normalized to the level of GAPDH mRNA. The results are presented as means \pm SD; ${ }^{*} \mathrm{P}<0.05$. (C and F) Western blot analysis of BAG3 and MMP2 expression. Total protein lysates from Ishikawa (C) and HEC108 (F) cells that were infected with a control (LTRH) or shBAG3 (BAG3 knockdown) vector were analyzed for BAG3 and MMP2 protein. $\beta$-actin served as a loading control.

vector encoding shRNA targeting BAG3 (shBAG3). In HEC108 cells, which exhibit substantial endogenous MMP2 expression, BAG3 knockdown (Fig. 2D and F, top blot) led to reduction in the expression of MMP2 mRNA and protein (Fig. 2E and F, middle blot). In Ishikawa cells, which express little MMP2, knocking down BAG3 (Fig. 2A and C, top blot) had no effect on MMP2 expression (Fig. 2B and C, middle blot).

BAG3 knockdown reduces cell motility and invasiveness. We next evaluated the effects of reducing endogenous BAG3 levels on cell motility and invasiveness. To assess motility, Transwell chambers treated with $10 \mu \mathrm{g} / \mathrm{ml}$ fibronectin were loaded with Ishikawa or HEC108 cells expressing either shBAG3 or a control sequence. As shown in Fig. 3A and B, BAG3 knockdown suppressed migration of both Ishikawa and HEC108 cells through the Transwell filter.

To assess the role of BAG3 in determining the invasiveness of tumor cells, Ishikawa or HEC108 cells expressing shBAG3 were placed on a Matrigel ECM layer in a Transwell chamber, and their rate of migration through the Matrigel was assayed. We found that BAG3 knockdown significantly reduced the invasiveness of the HEC108 cells, which strongly express MMP2 (Fig. 3D), but had little effect on the invasiveness of Ishikawa cells, which express little if any MMP2 (Fig. 3C).

Reducing BAG3 expression led to increases in miR29b levels in EAC cell lines. When we used real-time qRT-PCR to compare the levels of miR29b expression between Ishikawa and HEC108 cells, we observed that miR29b is more strongly expressed in Ishikawa than HEC108 cells (Fig. 4A). Moreover, knocking down BAG3 expression using shBAG3 enhanced miR29b expression in both Ishikawa and HEC108 cells (Fig. 4B and C).

miR-29b directly targets the MMP2 3'-UTR. To further investigate the role of miR-29b in determining the metastatic 
A
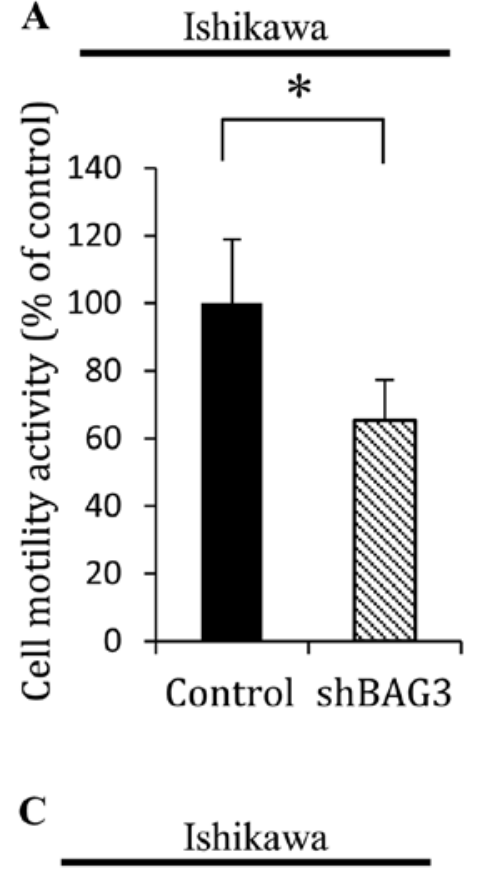

을

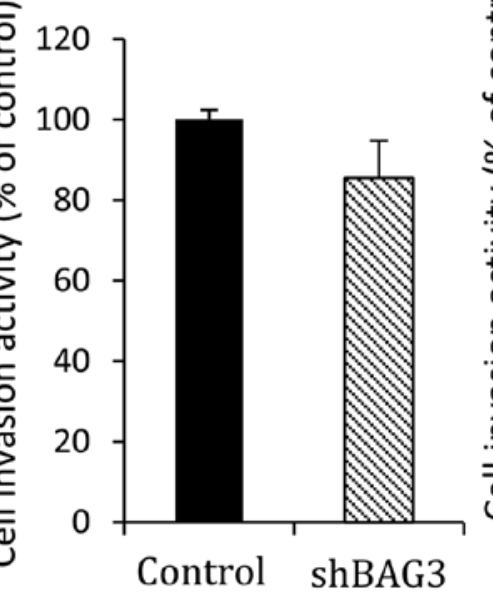

Control

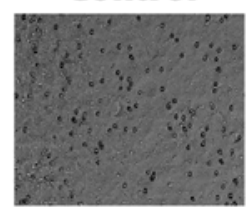

shBAG3

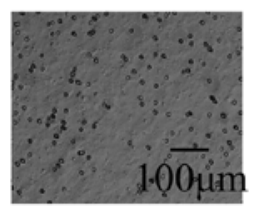

B
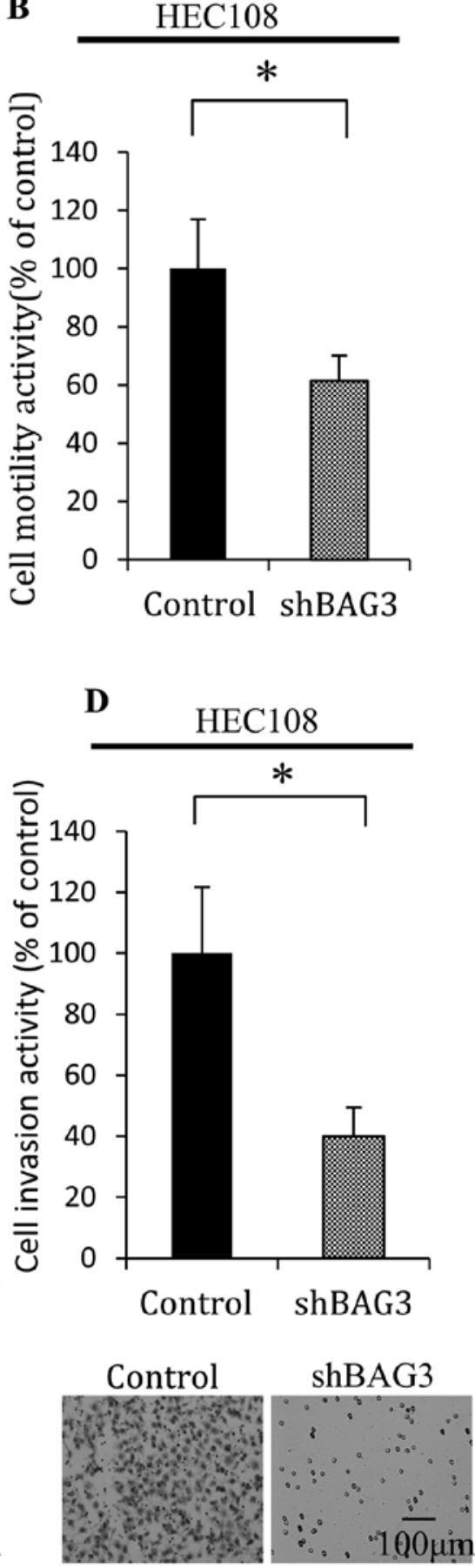

Figure 3. Effect of BAG3 knockdown on cell migration and invasion. (A and B) Migration of Ishikawa (A) and HEC108 (B) cells infected with a control (LTRH) or shBAG3 (BAG3 knockdown) vector was assessed in Transwell assays. Migration was allowed to proceed for $8 \mathrm{~h}$ prior to the assay. Bars depict means \pm SD. "P<0.05. (C and D) Invasion of Ishikawa (C) and HEC108 (D) cells that were infected with a control (LTRH) or shBAG3 (BAG3 knockdown) vector was assayed using Matrigel-coated Transwell chambers. Cells were allowed to invade the Matrigel for $48 \mathrm{~h}$ prior to the assay. Bars depict means $\pm \mathrm{SD}$. ${ }^{*} \mathrm{P}<0.05$.

potential of EAC cells, we transfected Ishikawa and HEC108 EAC cells with miRNA-29b mirVana miRNA mimic to augment miR-29b activity or with miRNA-29b mirVana miRNA inhibitor to diminish miR-29b activity. Using qRT-PCR, we observed that miR-29b expression was markedly increased in both Ishikawa and HEC108 cells transfected with miR-29b-mimic (Fig. 5A and B), and was diminished in both cell types transfected with miR-29b-inhibitor, though the effect appeared to be greater in HEC108 cells (Fig. 5C and D).
Then using a luciferase reporter system, we found that co-transfection of miR-29b-mimic significantly suppressed the activity of a reporter gene containing the MMP2 mRNA 3'-UTR (Fig. 5E). Conversely, inhibiting of endogenous miR-29b using a miR-29b-inhibitor increased the activity of the MMP2 reporter gene (Fig. 5F). In addition, MMP2 mRNA levels were lower than the control in Ishikawa and HEC108 cells transfected with miR-29b-mimic and were higher than the control in the cells transfected with miR-29b-inhibitor 

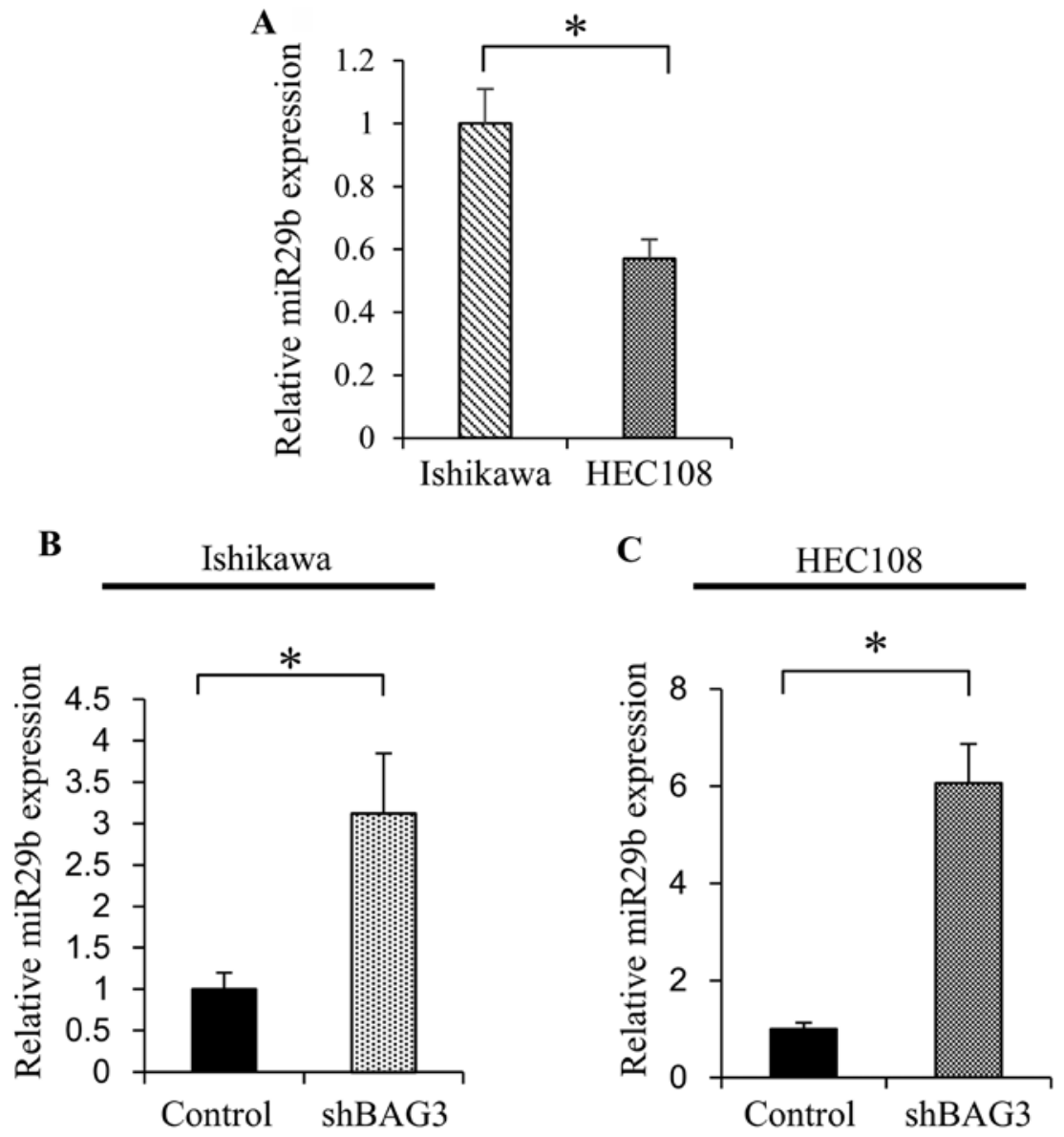

Figure 4. Expression of miR-29b in EAC cells. (A) Real-time qRT-PCR analysis of miR-29b expression in Ishikawa and HEC108 cells. Bars depict relative miRNA levels normalized to the RNU48 level (internal control). The results are presented as means \pm SD; ${ }^{*} \mathrm{P}<0.05$. (B and C) Real-time qRT-PCR analysis of miR-29b expression in Ishikawa (B) and HEC108 (C) cells that were infected with a control (LTRH) or shBAG3 (BAG3 knockdown) vector. Bars depict relative microRNA levels normalized to the RNU48 level (internal control). The results are presented as means \pm SD; ${ }^{*} \mathrm{P}<0.05$.

(Fig. 5G and H). Correspondingly, the levels of MMP2 protein were lower than the control in the HEC108 cells expressing miR-29b-mimic and higher than the control in the HEC108 cells expressing the miR-29b-inhibitor (Fig. 5J). In Ishikawa cells, by contrast, levels of MMP2 protein were slightly affected by miR29b-mimic or -inhibitor, as there was slight or no endogenous MMP2 expression in the Ishikawa cells (Fig. 5I). All things considered, these findings suggest miR-29b directly suppresses MMP2 expression through its binding to the MMP2 mRNA 3'-UTR.

Cell migration and invasion is inhibited by miR-29b in human $E A C$. We evaluated the effect of miR-29b in EAC cell motility and invasiveness using migration and Matrigel invasion assays after confirming the efficient transfection of miR-29bmimic or -inhibitor using qRT-PCR $48 \mathrm{~h}$ after transfection. The migration assays revealed that motility of both Ishikawa and HEC108 cells was significantly reduced by expression of miR-29b-mimic, as compared with the miR-control (Fig. 6A and C), and that motility was markedly increased by expression of the miR-29b-inhibitor (Fig. 6B and D).

Matrigel invasion assays demonstrated that the invasiveness of cells endogenously expressing MMP2 (HEC108) was significantly reduced by expression of miR-29b-mimic
(Fig. 6G), but was markedly increased by expression of miR-29b-inhibitor (Fig. 6H). On the other hand, expression of miR-29b-mimic or miR-29b-inhibitor had a slight, if any, effect in Ishikawa cells, which express a low level of MMP2 (Fig. 6E and F).

\section{Discussion}

Cell invasion is essential for cancer metastasis, which is the major cause of cancer mortality. It is well known that tumor metastasis is a complex process involving the loss of cell-cell adhesion and the gain of cell-matrix adhesion as well as the increase in the expression and activation of extracellular proteases that degrade the ECM, enabling cell migration through the space created. During this process, MMPs play a key role in catalyzing proteolytic degradation of the ECM (19). Among these enzymes, MMP2 catalyzed the degradation of type IV collagen and the overexpression of MMP2 has been observed in various types of cancers $(20,21)$. We found that the MMP2 expression was significantly weaker in the Ishikawa EAC cells (grade 1) than in the HEC108 EAC cells (grade 3). In addition, we demonstrated that reducing BAG3 or varying expression of miR-29b in HEC108 cells influenced both MMP2 expression and cell invasiveness, whereas Ishikawa cells, expressing 


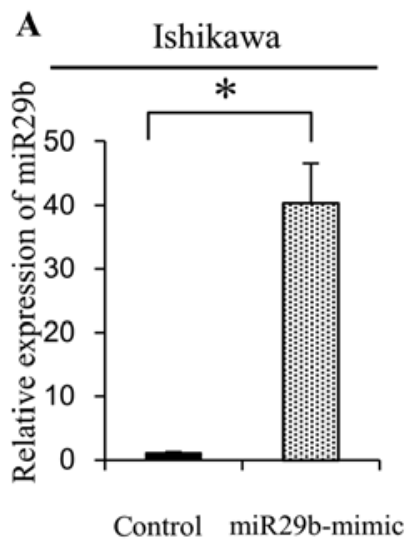

B

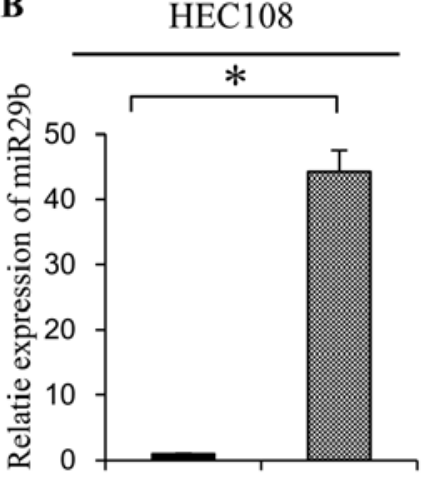

Control miR29b-mimic
C

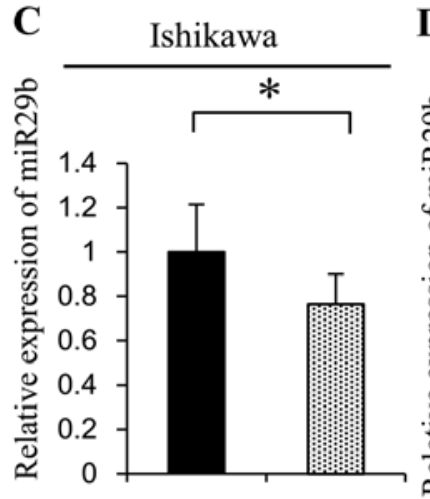

Control miR29b-inhibitor
D

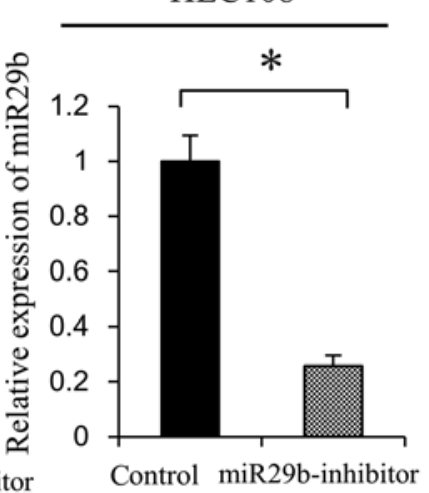

E

$*$
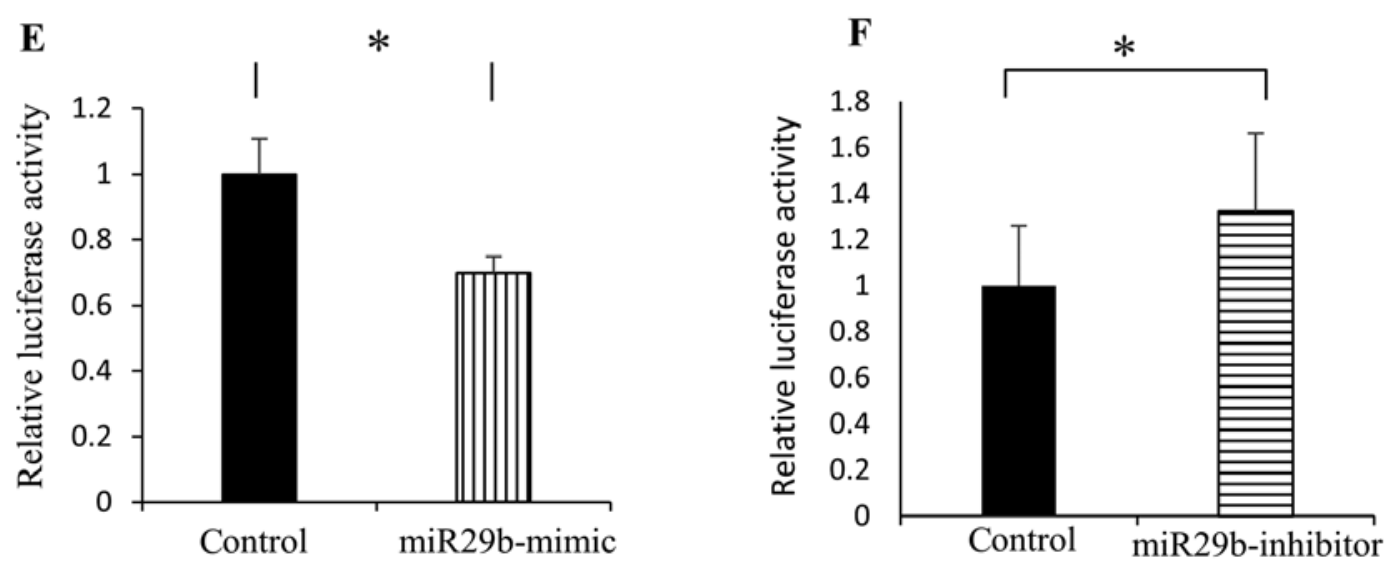

H

HEC108
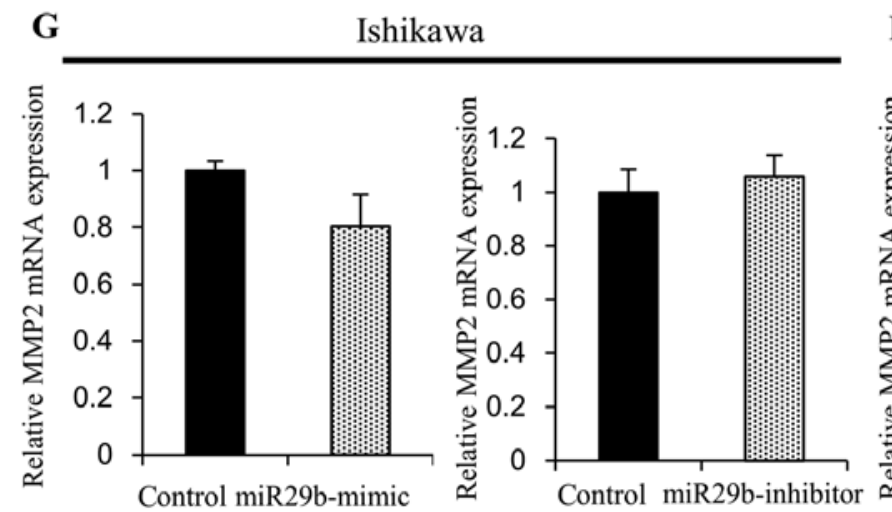

Ishikawa

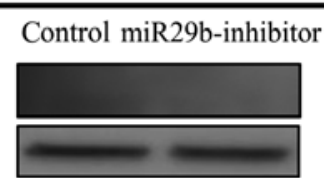

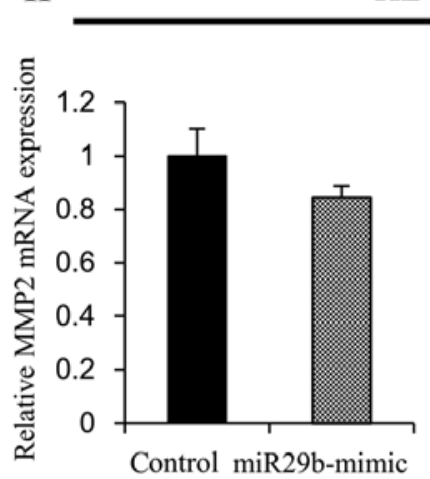

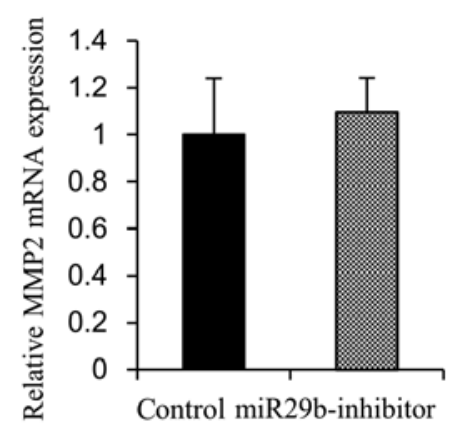

J

HEC108
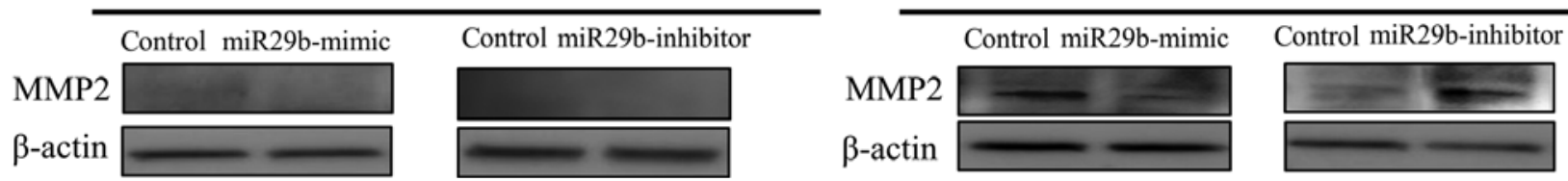

Figure 5. Exogenous expression of miR-29b inhibits MMP2 expression. (A-D) Ishikawa and HEC108 cells that were transfected with miR-29b mirVana miRNA mimic to augment miR-29b activity (A and B) or with miR-29b mirVana inhibitor to diminish miR-29b activity (C and D). Relative levels of miR-29b expression were then determined by using real-time qRT-PCR. Bars depict relative mRNA levels normalized to the RNU48 (internal control). The results are presented as means $\pm \mathrm{SD} ;{ }^{*} \mathrm{P}<0.05$. (E and F) EAC cells were co-transfected with miR-29b-mimic or its control (E) or with miR-29b-inhibitor (F) or its control, plus the Mcl-1mRNA 3'-UTR, which contains the target sites for miR-29b. Cells were collected $48 \mathrm{~h}$ after transfection and analyzed in luciferase assays. Bars depict luciferase activities normalized to the control levels. ${ }^{*} \mathrm{P}<0.05$. (G and H) Ishikawa (G) and HEC108 (H) cells that were transfected with miR-29b-mimic or its control, or with miR-29b-inhibitor or its control, were analyzed for MMP2 mRNA expression using real-time qRT-PCR. Bars depict relative mRNA levels normalized to the level of GAPDH mRNA. The results are presented as means \pm SD. (I and J) Ishikawa (I) and HEC108 (J) cells that were transfected with miR-29b-mimic or its control, or with miR-29b-inhibitor or its control, were analyzed for MMP2 protein expression using western blot analysis. As a loading control, the blot was reprobed with an anti- $\beta$-actin antibody.

much less MMP2 than HEC108 cells, showed little variation of MMP2 expression or cell invasiveness. These results indicate that MMP2 is a key mediator of EAC cell invasion.
BAG3 is known to be an important regulator of cancer cell migration, invasion and adhesion (7). A previous study from our group showed that BAG3 increases the invasiveness of 


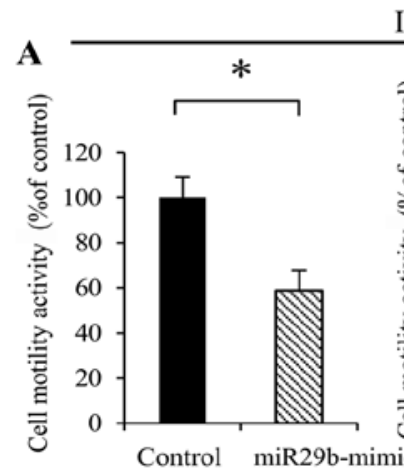

Ishikawa

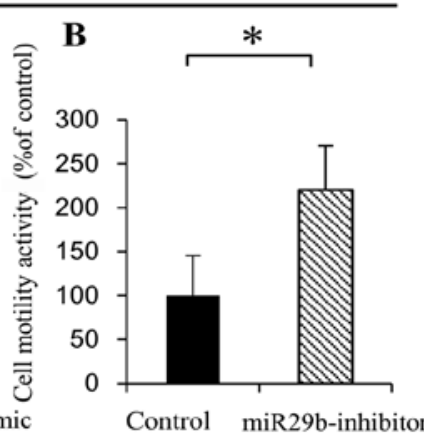

$\mathbf{E}$

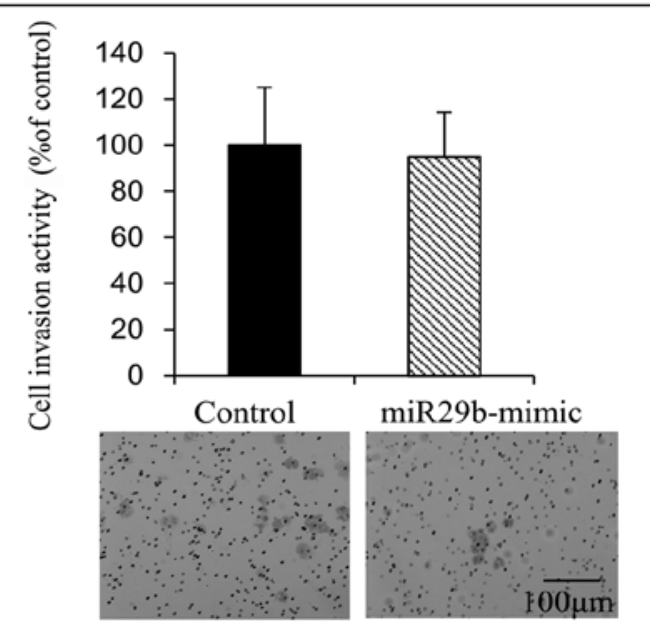

G

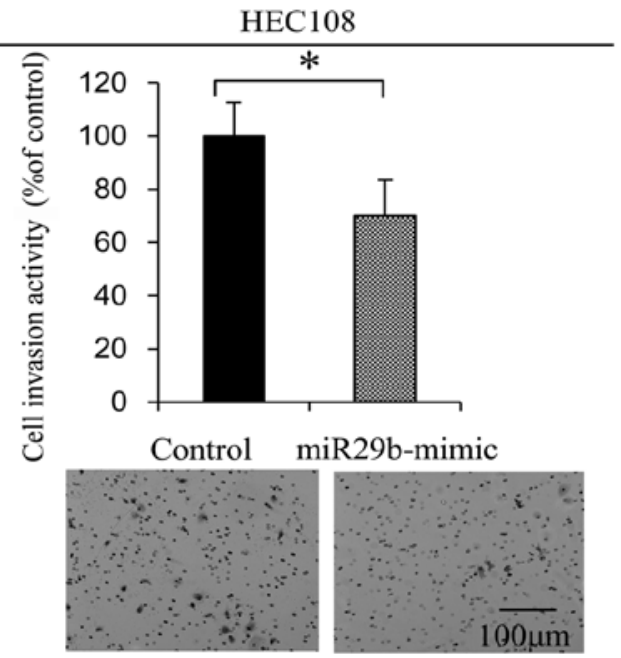

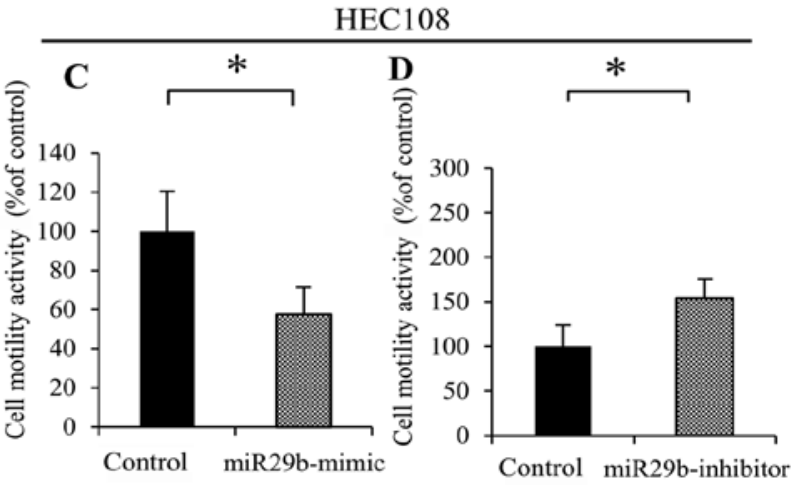

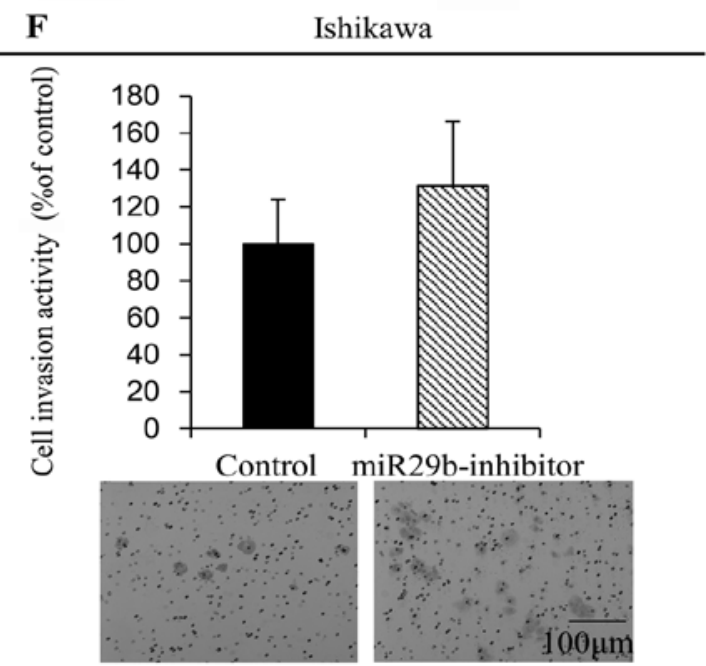

H

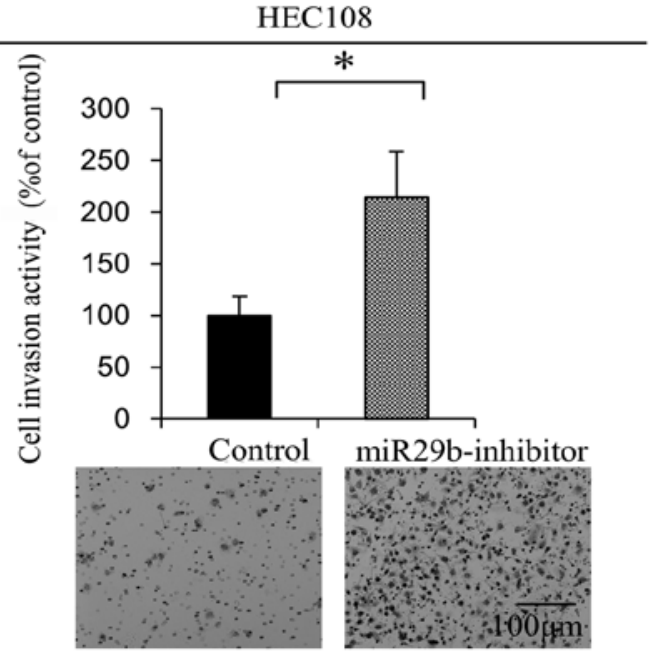

Figure 6. miR-29b expression decreases cancer cell motility and invasion. (A-D) Migration of Ishikawa (A and B) and HEC108 (C and D) cells transfected with miR-29b-mimic or its control (A and C), or with miR-29b-inhibitor or its control (B and D), was assayed using Transwell chambers. Migration was allowed to proceed for $8 \mathrm{~h}$ prior to the assay. (E-H) Invasion by Ishikawa (E and F) and HEC108 ( $\mathrm{G}$ and H) cells that were transfected with miR-29b-mimic or its control $(\mathrm{E}$ and $\mathrm{G})$ or with miR-29b-inhibitor or its control $(\mathrm{F}$ and $\mathrm{H})$ was assayed using Matrigel-coated Transwell chambers. Cells were allowed to invade the Matrigel for $48 \mathrm{~h}$ prior to the assay. Bars depict means $\pm \mathrm{SD}$. ${ }^{*} \mathrm{P}<0.05$.

cultured ovarian cancer cells by interacting with MMP2 (8). We also found that BAG3 knockdown leads to a reduction in the MMP2 expression, and that BAG3 binds to MMP2 to positively regulate the process of cell invasion. However, the molecular mechanism through which BAG3 enhances MMP2 expression is not known.

The expression of oncogenes is often regulated by miRNAs, small RNAs (21-25 nucleotides) that suppress gene expression by interacting with their mRNAs (22). Consequently, dysfunction or dysregulation of miRNAs can lead to the activation of oncogenes. Because miR-29b is known to directly suppress MMP2 expression in prostate cancer and hepatocellular carcinoma cells $(17,23)$ we examined the relationship between the expression of BAG3 and miR-29b. Our results show that BAG3 suppresses miR-29b expression in both Ishikawa and HEC108 cells. Moreover, luciferase reporter assays revealed that 
miR-29b directly targets MMP2 in EAC cells. It thus appears that inhibition of miR-29b correlates with enhanced MMP2 expression, as well as with increases in cell motility and invasiveness. Conversely, restoration of miR-29b reduced MMP2 expression and inhibited EAC cell motility and invasiveness. All things considered, these results suggest that inhibition of miR-29b by BAG3 leads to MMP2 overexpression and, in turn, increased EAC cell invasion.

The miR-29 family contains three members (miR-29a, -29b and $-29 c)$, which are highly similar and share the same seed sequence. Reduced expression of the miR-29 family miRNAs has been observed in several cancers, including acute myeloid leukemia, non-small cell lung cancer, cholangiocarcinoma and hepatocellular carcinoma. Moreover, reduced expression of miR-29b is significantly correlated with poorer overall survival and poorer progression-free survival in endometrial serous adenocarcinoma (13). However, miR-29 expression is not always diminished in cancer, as enhanced expression of miR-29 has been reported in both breast and colon cancer $(24,25)$. It will therefore be important to fully elucidate the mechanism by which miR-29 expression is regulated, which may enable miR-29 to serve as a therapeutic target. It has been reported that c-Myc negatively regulates $\mathrm{miR}-29 \mathrm{a} / \mathrm{b} 1$ expression at the transcription level (26). miR-29 expression also appears to be regulated via the NF- $\kappa$ B-YY1 pathway. Within that pathway, YY1 inhibits miR-29 transcription, whereas miR-29 blocks YY1 translation, and YY1 is also regulated by NF- $\mathrm{B}$ (27). In the present study, we found that BAG3 knockdown restores miR-29b expression in EAC cells, suggesting that BAG3 also inhibits miR-29b expression. Though further elucidation of molecular mechanism controlling the expression of miR-29b via BAG3 is necessary, understanding the involvement of BAG3 in regulating miR-29b expression may provide new insight into the process by which miR-29b activity is suppressed in EAC.

In conclusion, our results indicate that BAG3 suppresses expression of miR-29b. The reduction in miR-29b leads to enhanced expression of MMP-2, which in turn promotes cell migration and invasion. This scenario suggests that the BAG3-miR-29b-MMP2 pathway is a novel means by which BAG3 promotes cell invasion and contributes to EAC metastasis. It thus warrants further investigation.

\section{Acknowledgements}

We would like to thank Dr S. Takayama for providing a gene silencing vector (pLTRH1) specific for BAG3 and rabbit anti-BAG3 antibody.

\section{References}

1. DeSantis CE, Lin CC, Mariotto AB, Siegel RL, Stein KD, Kramer JL, Alteri R, Robbins AS and Jemal A: Cancer treatment and survivorship statistics, 2014. CA Cancer J Clin 64: 252-271, 2014.

2. Barrena Medel NI, Bansal S, Miller DS, Wright JD and Herzog TJ: Pharmacotherapy of endometrial cancer. Expert Opin Pharmacother 10: 1939-1951, 2009.

3. Einbond A and Sudol M: Towards prediction of cognate complexes between the WW domain and proline-rich ligands. FEBS Lett 384: 1-8, 1996.

4. Sudol M, Chen HI, Bougeret C, Einbond A and Bork P: Characterization of a novel protein-binding module - the WW domain. FEBS Lett 369: 67-71, 1995.
5. Doong H, Vrailas A and Kohn EC: What's in the 'BAG'? - A functional domain analysis of the BAG-family proteins. Cancer Lett 188: 25-32, 2002.

6. Doong H, Price J, Kim YS, Gasbarre C, Probst J, Liotta LA, Blanchette J, Rizzo K and Kohn E: CAIR-1/BAG-3 forms an EGF-regulated ternary complex with phospholipase C-gamma and Hsp70/Hsc70. Oncogene 19: 4385-4395, 2000.

7. Iwasaki M, Homma S, Hishiya A, Dolezal SJ, Reed JC and Takayama S: BAG3 regulates motility and adhesion of epithelial cancer cells. Cancer Res 67: 10252-10259, 2007.

8. Suzuki M, Iwasaki M, Sugio A, Hishiya A, Tanaka R, Endo T, Takayama S and Saito T: BAG3 (BCL2-associated athanogene 3) interacts with MMP-2 to positively regulate invasion by ovarian carcinoma cells. Cancer Lett 303: 65-71, 2011.

9. Bartel DP: MicroRNAs: Genomics, biogenesis, mechanism, and function. Cell 116: 281-297, 2004.

10. Nelson KM and Weiss GJ: MicroRNAs and cancer: Past, present, and potential future. Mol Cancer Ther 7: 3655-3660, 2008.

11. Banno K, Yanokura M, Kisu I, Yamagami W, Susumu N and Aoki D: MicroRNAs in endometrial cancer. Int J Clin Oncol 18: 186-192, 2013.

12. Devor EJ, Hovey AM, Goodheart MJ, Ramachandran S and Leslie KK: microRNA expression profiling of endometrial endometrioid adenocarcinomas and serous adenocarcinomas reveals profiles containing shared, unique and differentiating groups of microRNAs. Oncol Rep 26: 995-1002, 2011.

13. Hiroki E, Akahira J, Suzuki F, Nagase S, Ito K, Suzuki T, Sasano $\mathrm{H}$ and Yaegashi N: Changes in microRNA expression levels correlate with clinicopathological features and prognoses in endometrial serous adenocarcinomas. Cancer Sci 101: 241-249, 2010.

14. Garzon R, Heaphy CE, Havelange V, et al: MicroRNA 29b functions in acute myeloid leukemia. Blood 114: 5331-5341, 2009.

15. Sugio A, Iwasaki M, Habata S, Mariya T, Suzuki M, Osogami H, Tamate M, Tanaka R and Saito T: BAG3 upregulates Mcl-1 through downregulation of miR-29b to induce anticancer drug resistance in ovarian cancer. Gynecol Oncol 134: 615-623, 2014

16. Mott JL, Kobayashi S, Bronk SF and Gores GJ: miR-29 regulates Mcl-1 protein expression and apoptosis. Oncogene 26: 6133-6140, 2007.

17. Fang JH, Zhou HC, Zeng C, Yang J, Liu Y, Huang X, Zhang JP, Guan XY and Zhuang SM: MicroRNA-29b suppresses tumor angiogenesis, invasion, and metastasis by regulating matrix metalloproteinase 2 expression. Hepatology 54: 1729-1740, 2011.

18. Homma S, Iwasaki M, Shelton GD, Engvall E, Reed JC and Takayama S: BAG3 deficiency results in fulminant myopathy and early lethality. Am J Pathol 169: 761-773, 2006.

19. Stetler-Stevenson WG, Aznavoorian S and Liotta LA: Tumor cell interactions with the extracellular matrix during invasion and metastasis. Annu Rev Cell Biol 9: 541-573, 1993.

20. Ogasawara S, Yano H, Momosaki S, Nishida N, Takemoto Y, Kojiro $S$ and Kojiro M: Expression of matrix metalloproteinases (MMPs) in cultured hepatocellular carcinoma (HCC) cells and surgically resected HCC tissues. Oncol Rep 13: 1043-1048, 2005.

21. Giannelli G, Bergamini C, Marinosci F, Fransvea E, Quaranta M, Lupo L, Schiraldi O and Antonaci S: Clinical role of MMP-2/ TIMP-2 imbalance in hepatocellular carcinoma. Int J Cancer 97: 425-431, 2002

22. Negrini M, Ferracin M, Sabbioni S and Croce CM: MicroRNAs in human cancer: From research to therapy. J Cell Sci 120: 1833-1840, 2007.

23. Steele R, Mott JL and Ray RB: MBP-1 upregulates miR-29b that represses Mcl-1, collagens, and matrix-metalloproteinase- 2 in prostate cancer cells. Genes Cancer 1: 381-387, 2010.

24. Wang Y, Zhang X, Li H, Yu J and Ren X: The role of miRNA-29 family in cancer. Eur J Cell Biol 92: 123-128, 2013.

25. Gebeshuber CA, Zatloukal K and Martinez J: miR-29a suppresses tristetraprolin, which is a regulator of epithelial polarity and metastasis. EMBO Rep 10: 400-405, 2009.

26. Mott JL, Kurita S, Cazanave SC, Bronk SF, Werneburg NW and Fernandez-Zapico ME: Transcriptional suppression of miR-29b-1/miR-29a promoter by c-Myc, hedgehog, and NF-kappaB. J Cell Biochem 110: 1155-1164, 2010.

27. Wang H, Garzon R, Sun H, et al: NF-kappaB-YY1-miR-29 regulatory circuitry in skeletal myogenesis and rhabdomyosarcoma. Cancer Cell 14: 369-381, 2008. 